\title{
Introducing new perspectives in radioguided intervention
}

\author{
Renato A. Valdés Olmos ${ }^{1,2} \cdot$ Sergi Vidal-Sicart ${ }^{3}$
}

Received: 24 February 2016/Accepted: 28 March 2016/Published online: 11 April 2016

(C) Italian Association of Nuclear Medicine and Molecular Imaging 2016

The incorporation of the sentinel lymph node (SLN) procedure to the management of cutaneous melanoma and breast cancer in the 1990s reinforced the importance of radioguided surgery as one of the growing roles of Nuclear Medicine. From the beginning, this field involved several aspects characterized by a remarkable collaboration with other clinical specialties as well as a close cooperation with other more technical disciplines allied to Nuclear Medicine to develop new tracers and devices. The multidisciplinarity and the clinical usefulness of these procedures resulted in an immediate and long-term benefit for the patient. Today, radioguided procedures imply a minimal morbidity in comparison with classical surgical approaches, with the potential of equal or better staging and regional control. In the present millennium, hybrid imaging has become possible and enabled to depict targets for radioguided biopsy in an anatomical environment. This interventional imaging facilitated the generation of three-dimensional roadmaps for surgeons necessary to intraoperatively localize and remove not only SLNs but also radiotracer-avid lesions with a diagnostic and/or therapeutic intention.

Following an initiative of the International Atomic Energy Agency (IAEA) a few years ago the concept

Renato A. Valdés Olmos

R.A.Valdes_Olmos@lumc.nl

1 Nuclear Medicine Section and Interventional Molecular Imaging Laboratory, Department of Radiology, Leiden University Medical Centre, PO Box 9600, 2300 RC Leiden, The Netherlands

2 Department of Nuclear Medicine, Antoni van Leeuwenhoek Hospital, The Netherlands Cancer Institute, Amsterdam, The Netherlands

3 Nuclear Medicine Department, University Hospital Clinic, Barcelona, Spain
"Guided intraOperative Scintigraphic Tumour Targeting" (GOSTT) was introduced to encompass the whole spectrum of basic and advanced nuclear medicine procedures required to provide a roadmap for radioguided surgery [1]. The GOSTT concept is based on both preoperative imaging and intraoperative radioguided target tissue localization (Fig. 1). The SLN procedure is probably the most basic approach for GOSTT and includes the local administration of a radiotracer into or near the tumour which enables the visualization of lymphatic drainage and the identification of SLNs by means of lymphoscintigraphy. This classical SLN biopsy has been adopted as procedure in the surgical guidelines for melanoma and breast cancer by using preoperative lymphoscintigraphy and intraoperative dyes and handheld gamma probes. Nowadays, this approach has been extended to other malignancies such as head and neck cancers, gynecological cancers (vulvar cancer, cervix cancer, endometrial cancer), urological malignancies (prostate cancer, bladder cancer, penile carcinoma and others), gastrointestinal cancers, lung cancer, thyroid carcinoma, and others with different success and different technical requirements $[2,3]$.

\section{Current GOSTT approaches for interventional imaging and radioguided surgery}

Excepting SLN biopsy, the area with the greatest potential for GOSTT probably is the radioguided procedures for excisional biopsy of target lesions with diagnostic or therapeutic purposes. This potential is based on the detection by SPECT/CT and PET/CT of occult lesions with uptake of a tumour-seeking radiotracer. This may lead to subsequent radioguided intervention, which can be based on different approaches. In this respect at present, three 
Fig. 1 On the left, schematic illustration of preoperative interventional imaging showing sentinel lymph nodes in the right axilla and groin on SPECT/CT as well as a ${ }^{18} \mathrm{~F}$ FDG avid metastasis in the left groin on PET; for both examples melanoma was located on the back. On the right, compilation of some current possibilities for intraoperative and preoperative radioguided intervention

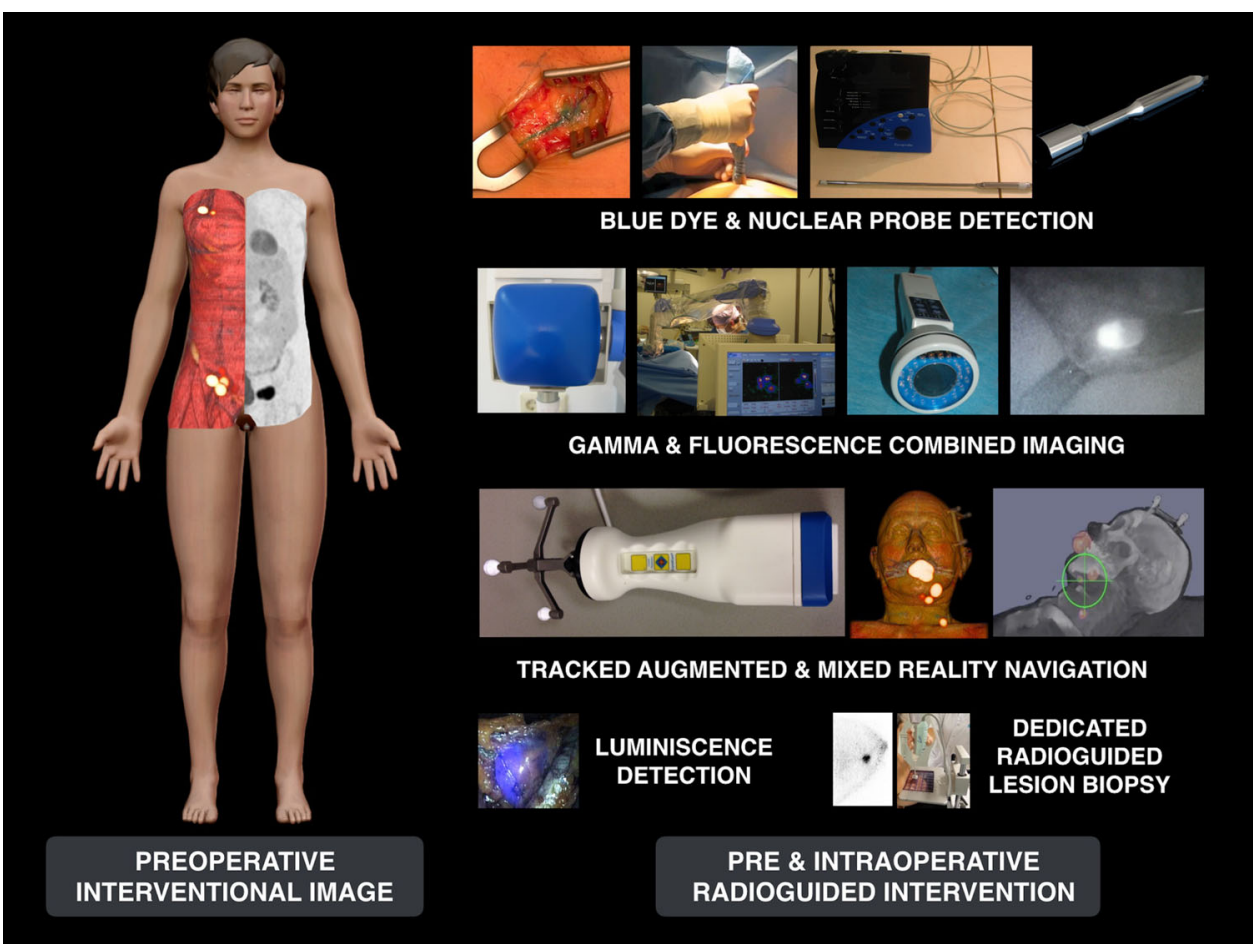

GOSTT working models can be delineated in relation to radiotracer administration: intralesional without tracer migration for occult lesion localization, intralesional with tracer migration for occult lesion localization and sentinel lymph node biopsy, and systemic to enable excisional biopsy of primary lesions and recurrences as well as isolated regional and distant metastases.

Direct intralesional injection of a radiotracer without migration enables subsequent excision of the targeted tissue; this approach was originally validated using ${ }^{99 \mathrm{~m}} \mathrm{Tc}-$ MAA in non-palpable breast cancer patients. To accomplish concentricity in the distribution into the tumour the radiotracer needs to be injected as centrally as possible in the lesion. During excision, which can be performed on the same day or on the next day, the gamma probe is introduced through a small skin incision that ensures an optimal cosmetic outcome without the topographic constraints determined by the classical intralesional markers such as wire. Lately this Radioguided Occult Lesion Localization (ROLL) procedure has been extended to other lesions such as solitary pulmonary nodules, involved lymph nodes, bone- and soft tissue metastases, and others. Also, the ROLL technique demonstrated excellent results and a low percentage of re-interventions for remaining tumoral tissue in breast cancer patients. A variant of the ROLL procedure is the use of a capsulated source of radiation to be implanted into the targeted lesion to enable its subsequent excision. In this sense, the introduction of Radioguided Seed Localization (RSL) using ${ }^{125} \mathrm{I}$-seeds has gained in acceptance in the surgical world due to improved patient comfort, increased ease of surgical scheduling, decreased risk for displacement and subsequent enlargement of the specimen. RSL performed before surgery is a good alternative to wire or ROLL localization, allowing flexibility in scheduling and improving workflow in imaging procedures and surgery. Furthermore, thanks to new clinical scenarios, like the possibility to perform resection of residual primary tumours and sentinel lymph node biopsy following primary systemic therapy, RSL might play a role to accurately ascertain the treatment results. For example, RSL procedure can serve for marking lymph nodes containing metastasis before primary systemic therapy. The response to treatment can be analysed afterwards localizing the marked node with radioguided surgery. In breast cancer, this method can avoid an aggressive axillary treatment in the axilla if primary systemic therapy has downstaged the previously involved node.

The second GOSTT model concerns intralesional radiotracer administration with subsequent partial migration to regional lymph nodes. To accomplish this, usually colloid particles smaller than those used for ROLL are injected. Basic work based on ex vivo lymph node measurements carried out 20 years ago in the context of validation of the SLN procedure demonstrated that when ${ }^{99 \mathrm{~m}} \mathrm{Tc}$-nanocolloid is administered into a breast tumour, only $2-3 \%$ of the activity migrates to the axillary SLNs. This property facilitates the combination of ROLL with sentinel lymph node biopsy in breast conserving surgery 
following single injection of the radiotracer and is known as SNOLL (Sentinel Node and Occult Lesion Localization).

Radioguided surgery related to the systemic administration of a tracer is the GOSTT working model based on the detection by SPECT/CT and PET/CT of target lesions with subsequent indication for resection for diagnostic or therapeutic purposes. The indication may be closely related to suspected cancer recurrence with elevation of tumour markers during follow-up. For example, PET/CT in combination with a hand-held PET probe has gained expansion with the improvement of intraoperative radioguidance using different tracers and dedicated PET probes to detect the high-energy gamma photons or $\beta^{+}$particles. For SPECT/CT a similar approach is possible. For instance, ${ }^{99 \mathrm{~m}}$ Tc-sestamibi has been extensively validated for parathyroid surgery, and radiolabeled agents with receptormediated uptake e.g., neuroendocrine tumours. Classical approaches of this GOSTT concept can also be found in the radioguided excision of isolated bone metastasis or bony lesions due to the accumulation of bone-seeking agents, and radioiodine avid recurrences/metastases from differentiated thyroid carcinoma.

\section{Improving synergy of SPECT/CT and PET/CT with portable imaging devices in the operating theatre}

The anatomical localization of SLNs or tracer avid lesions has transformed SPECT/CT and PET/CT from a staging modality to one providing imaging for radioguided interventions. Surgeons can now use the provided anatomical landmarks to retrieve the detected target lesions during operation. This evolution to a 3D roadmap after the incorporation of these hybrid molecular modalities has helped to transform the GOSTT paradigm to one of "see, open and recognize" [4]. This approach has been reinforced with the synergy of preoperative SPECT/CT and PET/CT with intraoperative portable imaging technologies. Dedicated intraoperative portable gamma cameras have increasingly been incorporated in radioguided surgery in the last decade. Real-time imaging with an intraoperative gamma camera provides a larger field of view than a gamma probe can cover, and visual assistance in localization and verification of resection of the targeted tissue. Its position can be adjusted to also show sentinel nodes near the radiotracer injection site or to distinguish between two radiolabeled tissues, which can easily be overlooked by using a conventional handheld gamma probe. Examples can be found in the intraoperative localization of SLNs or parathyroid adenomas in complex or difficult anatomical areas like the head and neck. These devices have been particularly helpful to depict SLNs at a distance of at least $3 \mathrm{~mm}$ from the injection site when equipped with a highresolution pinhole collimator. Moreover, to overcome the lack of anatomical information the prototypes of a new generation of portable gamma cameras have been upgraded with an optical module for fused optical/scintigraphic imaging resulting in improved anatomical localization.

Another important possibility for the use of SPECT/CT and PET/CT findings in the surgical act is the intraoperative real-time generation of virtual elements. These elements can be added to the surgical environment in the context of an augmented reality protocol. Augmented reality approach can be considered as a part of mixed reality, but it is also an independent modality, which has been applied in radioguided surgery mainly after the introduction of the freehand SPECT technology based on a tracked gamma probe suitable for 3D image generation. This device has been the basis for augmented reality experiences in SLN procedures in breast cancer, oral cancer, melanoma as well as for surgery of parathyroid adenomas. The same freehand SPECT technology may be considered a helpful tool in the transference of SPECT/CT, and potentially PET/CT, to the operating room using mixed reality protocols. The concept of mixed reality was introduced by Milgram and Kishino in 1994 and concerns the merging of real and virtual elements to produce new environments. More related to the GOSTT approach, with the use of a tracked protocol today it has become possible to transfer the virtual SPECT/CT images to the operating room, so that a merged environment can be created for adequate surgical navigation [5]. This approach has been found to be feasible in areas of rigid anatomy like the retroperitoneal space, for instance when used to navigate towards the prostate and SLNs in robot-assisted prostatectomy. However, more difficulties are probably expected for "non-rigid" anatomical areas. Recently, freehand SPECT in combination with a mobile handheld gamma camera has been validated. As will be reviewed in this issue, the freehand SPECT technology has also been used to generate intraoperative $3 \mathrm{D}$ navigation in breast-preserving cancer surgery for single or multiple ${ }^{125} \mathrm{I}$-seeds in RSL as well as for ROLL.

\section{Allied technologies}

In the recent evolution of GOSTT some novel non-nuclear medicine technologies have been added to the conventional portable devices to improve intraoperative detection of target lesions. An example is the combined detection of SLNs using portable gamma cameras and near-infrared cameras (NIR). The introduction of fluorescent agents to the SLN procedure makes it possible today to use this 
modality in addition to the gamma ray detection. Although various groups have validated intraoperative fluorescent imaging for SLN independently, the limitations of this approach to generate adequate preoperative lymphatic mapping has led to search for alternative approaches based on the combination of both techniques. To accomplish this, two approaches can be delineated: the first one is based on the use of two separate agents, the radioactive tracer administered preoperatively and the fluorescent agent injected intraoperatively. The second approach is based on the use of a hybrid tracer combining radioactivity and fluorescence in one signature; this approach enables nuclear physicians to perform preoperative lymphatic mapping including SPECT/CT and subsequently surgeons in the operating room to combine portable gamma detection with fluorescence NIR-camera imaging. On a clinical basis, the most extensively validated hybrid tracer is currently ICG- ${ }^{99 \mathrm{~m}}$ Tc-nanocolloid which showed $100 \%$ reproducibility when compared with the parental $99 \mathrm{~m} \mathrm{Tc}$ nanocolloid, and subsequently has been introduced for SLN biopsy in melanoma, breast cancer, oral malignancies and urogenital cancer. This hybrid approach combining radioguidance technology with fluorescence, whereby the strengths of the individual technologies remain preserved, and are complemented by the strengths of the other modalities, not only may improve surgical logistics, but also provides perspective for more complex radioguided procedures in the future.

Also optical imaging can be used in parallel or combined with nuclear imaging in clinical approaches and probably dedicated-tracers with an optical signature will be required to facilitate this approach. A recent example, Cerenkov imaging utilizes light emission provoked by $\beta$ particles crossing through a medium. This technique can be applied to known PET tracers such as ${ }^{18}$ F-FDG. However, the low photon flux and an unfavourable wavelength are major limiting factors, making the technique difficult to immediately be applied in clinical settings. New efforts are being developed in this field to optimize this technology enabling a wide gamma of applications.

\section{Other radioguided biopsy possibilities}

Against the GOSTT background it is possible to visualize an evolution of the concept of radioguided surgery to a broader one of radioguided intervention. A number of novel applications are related to the incorporation of dedicated molecular imaging for biopsy. Two examples to be discussed in this issue of Clinical and Translational Imaging are related to breast cancer. Following systemic administration of ${ }^{18} \mathrm{~F}$-FDG breast lesions with uptake of this radiotracer can be biopsied with high precision using a dedicated PET device able to provide depth information. A similar approach is currently possible using a dedicated gamma camera and a specific biopsy device to sample breast lesions with uptake of ${ }^{99} \mathrm{~m}$ Tc-sestamibi. Possible clinical indications for both modalities concern radiologically occult breast lesions and a more accurate tumour sampling in patients with locally advanced breast cancer scheduled to receive neoadjuvant chemotherapy.

\section{Future GOSTT scenarios}

Current GOSTT advances open windows to new scenarios concerning clinical indications and modern technologies. In addition to the existing devices and modalities for radioguided surgery some future possibilities and scenarios can be summarized as follows:

1. Development of new systemic radiotracers for interventional imaging. The best example for this issue is illustrated by the recent introduction of ${ }^{68} \mathrm{Ga}-\mathrm{PSMA}$ for prostate cancer and its interventional use in resection of lymph node metastases. The pairing of this PET tracer to ${ }^{111}$ In-PSMA enables preoperative SPECT/CT imaging and subsequent radioguided surgery based on standard gamma ray detection.

2. ROLL and SNOLL. The ROLL model will probably be extended to resection of recurrences, regional lymph node metastases or isolated subclinical metastasis. The SNOLL approach is of potential use in other malignancies like thyroid, lung and stomach.

3. SLN guided lymph node template resection. This model is currently discussed for high-risk prostate cancer. The use of radiocolloids to guide SLN identification can be of help to optimize separate injection of fluorescent agents to guide lymph node resection in the basin of the SLN.

4. RSL following neoadjuvant chemotherapy. In analogy with the setting for locally advanced breast cancer and based on the practical advantages due to the long half life of iodine-125, this approach could enable seed implantation before chemotherapy and excision of residual tumour or lymph node metastasis after treatment in other local advanced malignancies scheduled for neoadjuvant therapy.

5. Mixed-reality navigation. The use of new sensor technology for tracking will probably help to transfer SPECT/CT, PET/CT, and PET/MRI with improved resolution to operation rooms, in order to facilitate image guided navigation to target regions.

6. Hybrid detection procedures. Portable imaging devices as gamma cameras will probably be integrated with other modalities as NIR cameras. Also, the integration 
of Cerenkov luminescence devices will extend the intraoperative applicability of PET tracers. Similarly, tracked acquisition of gamma ray devices and realtime ultrasound will enable fusion of these modalities for improved targeted fine needle aspiration or biopsy of SLN or tumour lesions.

7. Robot-assisted procedures with integrated radioguided navigation. In a similar approach as the one used for prostate cancer the fluorescence and probably the radioactive imaging will be integrated to robot-assisted procedures in other pelvic malignancies. Development of novel accessory devices like drop-in gamma probes for insertion and residing inside the abdominal cavity enabling a robotic gripper; this approach will make identification of SLNs and tumour lesions during laparoscopic surgery more flexible by improving detector accessibility and geometry.

8. In the context of the assessment of therapy response, the incorporation of specific tracers for dedicated radioguided biopsy will improve tumour characterization concerning gene expression profiles and molecular subtypes.

In this issue of Clinical and Translational Imaging several reviews about the different approaches and future directions in radioguided surgery as described above will show the current possibilities and the more recent investigational applications that has a potential for the upcoming clinical uses in this field.

\section{Compliance with ethical standards}

Informed consent This article does not contain any studies with human or animal subjects performed by the any of the authors.

Conflict of interest Renato A. Valdés Olmos and Sergi Vidal-Sicart declare no conflict of interest.

\section{References}

1. Zaknun JJ, Giammarile F (2012) Valdés Olmos RA, Vidal-Sicart $\mathrm{S}$, Mariani G. Changing in radioguided surgery and intraoperative imaging: the GOSTT concept. Eur J Nucl Med Mol Imaging 39:1-3

2. International Atomic Energy Agency (IAEA), ed. Guided Intraoperative Scintigraphic Tumour Targeting (GOSTT): Implementing advanced hybrid molecular imaging and non-imaging probes for advanced cancer management. Vienna: IAEA 2014

3. Saad Z, Buscombe J (2015) Sentinel lymph node: established and new areas of use. Clin Transl Imaging. 3:225-236

4. Valdés Olmos RA, Vidal-Sicart S, Giammarile F, Zaknun FF, Van Leeuwen FW, Mariani G (2014) The GOSTT concept and hybrid mixed/virtual/augmented reality environment radioguided surgery. Q J Nucl Med Mol Imaging 58:207-215

5. Waelkens $\mathrm{P}$, van Oosterom MN, van den Berg NS, Navab N, van Leeuwen FWB (2016) Surgical navigation: an overview of the state-of-the-art clinical applications. In: Herrmann K, Nieweg OE, Povoski SP (eds) Radioguided surgery-current applications and innovative directions in clinical practice. Springer, Heidelberg 